\title{
FATIGUE PERFORMANCE ANALYSIS AND EXPERIMENTAL STUDY OF STEEL TRUSSES INTEGRAL JOINT BASED ON MULTI-SCALE FEM
}

\author{
Huili Wang1 ${ }^{1,2^{*}}$ - Hao Gao ${ }^{1}$ - Sifeng Qin ${ }^{3}$
}

${ }^{1}$ Institute of Bridge Engineering, Dalian University of Technology, Dalian

${ }^{2}$ State Key Lab of Subtropical Building Science, South China University of Technology, Guangzhou

${ }^{3}$ Materials Fracture Mechanics, Dalian University Research Center for Numerical Test, Dalian

\begin{tabular}{l}
\hline ARTICLE INFO \\
\hline Article history: \\
Received: 14.7 .2015$. \\
Received in revised form: 17.10 .2015$. \\
Accepted: 19.10 .2015$. \\
\hline Keywords: \\
Fatigue \\
Experiment \\
Multi-scale FEM
\end{tabular}

\section{Introduction}

The multi-scale phenomenon is a frontier and hot research issue which belongs to the multidisciplinary research categories, including mathematics, mechanics, material, biology and so

\begin{abstract}
:
The fatigue performance of steel truss integral joint is analyzed based on multi-scale FEM. Numerical results are verified with experimental ones. The fatigue performance of steel truss integral joint is analyzed using a sea-crossing suspension bridge as its background. The 1:2 scale model fatigue test is taken. After 2e6 time load loops, the stress situation of integral joint is stable and fatigue performance is satisfactory for requirements. The multi-scale FEM, whose main researching part adopts three-dimensional elements while the connecting component adopts the common beam element, can reduce the computational cost. The connection elements are used to connect the beam elements and threedimensional elements. The connection element has two joints, one of which has six degrees of freedom, three translation degrees of freedom and three rotational degrees of freedom, all degrees of freedom being coupled. The multi-scale FEM and the experimental one yield very close results The multi-scale FEM can provide an accurate simulation of the main research part and ensure the fatigue life forecast so as to simplify the calculation. This method is high efficient and feasible. The multi-scale FEM provides a new and reliable method for the fatigue performance of structure analysis.
\end{abstract}

on. The essential idea of multi-scale FEM may be a frontier and hot research issue and the primary function of a non-polynomial form which Babuska [1] proposed in 1994. In 1997, Hou [2] expanded this essential idea and proposed the multi-scale FEM explicitly. Chen [3] studied the ultra-sample

\footnotetext{
* Corresponding author. Tel.: 086-0411-84707232; Fax: 086-411-84706275

E-mail address: wanghuili@dlut.edu.cn
} 
multi-scale FEM. Allaire [4] studied the multi-scale FEM homogenized plan so that by conforming partial thin grid and the overall situation thick grid he obtained the primary multi-scale function. Aarnes [5] developed graduation multi-scale FEM based on the mix FEM. Zhang [6] developed the space and time multi-scale FEM. At present, multiscale FEM research mainly concentrates on the multi-scale analysis of material behavior. The traditional single scale structure simulation is unable to analyze the large-scale civil engineering structure fatigue damage. Based on the material multi-scale analysis method, multi-scale FEM is adapted for complex structure analysis and damage assessment ${ }^{[7-8]}$. The crack problem exists generally in each material and engineering structure. There is grid restructuring and a large number of freedom degrees are combined with traditional FEM to carry on fracture growth simulation. Since traditional single scale structure simulation is unable to analyze large-scale civil engineering structure fatigue damage, multi-scale FEM is widely used in the research to question fracture growth. With multi-scale FEM, Sun [9] studied mechanical engineering characteristics of crack process area. Qu [10] studied steel fatigue cracking structure expansion under dynamic load based on multi-scale FEM.

Steel trusses integral joint as a whole is achieved by welding the chord into the gusset plate. The joint is the spaced welding structure and therefore, it is very concerned with the fatigue performance. Scholars have done a lot of work in the field of fatigue cumulative damage, and put forward some calculation models and fatigue cumulative damage rules, such as linearity fatigue cumulative damage theory [11], bilinear fatigue cumulative damage theory [12], non-linear fatigue cumulative damage theory [13], probability fatigue cumulative damage theory [14] and so on. At present, the fatigue problem is investigated with FEM and model test. The FEM is based on linear fatigue cumulative damage theory and linear elastic fracture mechanics. The traditional single scale structure simulation is unable to analyze the large-scale civil engineering structure fatigue damage, to forecast the fatigue life and crack development process [15]. However, if solid elements are used for the whole structure, this complex calculation can be liable to make mistakes. The multi-scale FEM can reduce the computational cost so that the main researching part adopts solid elements while other parts adopt the common beam element. The traditional single scale structure simulation is unable to analyze the large-scale civil engineering fatigue damage structure. Verified by the experimental data, this method is highly efficient and feasible.

\section{Multi-scale FEM}

The micro-scales elements are adopted for an integral joint part. The common elements are employed for other components. The integral joint part uses solid elements while a connecting component uses the ordinary beam elements. The calculation is simplified and method yields high precision. The connection elements are used to connect beam elements and solid elements ${ }^{[16]}$. The connection element has two joints, one of which has six degrees of freedom, three translation degrees of freedom and three rotational degrees of freedom, and all degrees of freedom are coupled.

The connection element is a key issue in multi-scale FEM. For a non-restraint connection element (Fig. 1), the relative displacement between nodes $i$ and $j$ is as follows:

$u_{n}=e_{n}^{i}\left(x^{j}-x^{i}\right)-E_{n}^{i}\left(X^{j}-X^{i}\right)(n=1,2,3)$

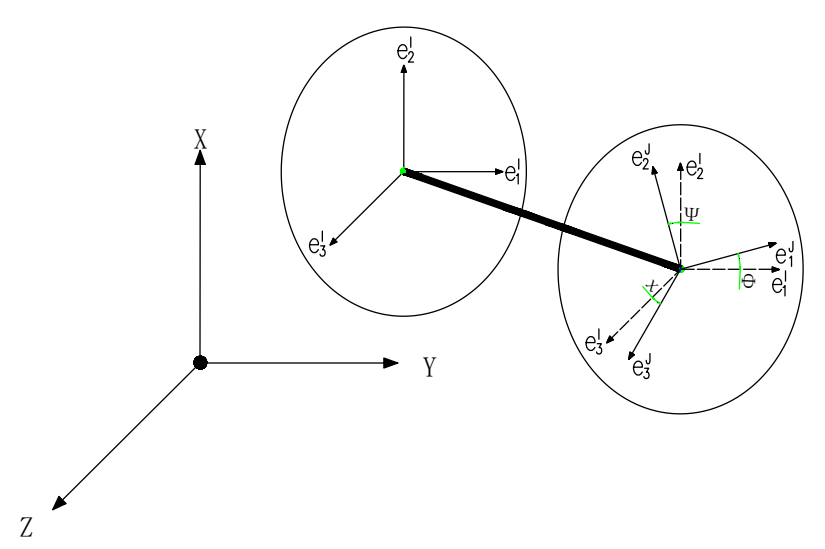

Figure 1. Contact element geometry calculation diagram

In the formula, $u_{n}$ is translation component under a local coordinate system. $e_{n}^{i}$ is a local coordinate axis for three node locations $i . E_{n}^{i}$ is $e_{n}^{i}$ axis corresponding component after node $i$ moving. For node $i$ and $j, x^{i}$ and $x^{j}$ is position vector in the 
current structure. $X^{i}$ and $X^{j}$ is position vector in reference configuration. Relative rotation relations between node $i$ and $j$ are as follows:

$$
\begin{aligned}
& \phi=-\arctan \left(\frac{e_{2}^{i} \cdot e_{3}^{j}}{e_{3}^{i} \cdot e_{3}^{j}}\right) \\
& \varphi=-\arcsin \left(e_{1}^{i} \cdot e_{3}^{j}\right) \\
& \chi=-\arctan \left(\frac{e_{1}^{i} \cdot e_{2}^{j}}{e_{1}^{i} \cdot e_{1}^{j}}\right)
\end{aligned}
$$

$\phi, \varphi$ and $\chi$ are first, second and third Cardan angles localized under the local coordinate system. For the unconstrained connection element, we use the following definitions to commutate relative displacement.

$$
\begin{aligned}
& u_{n}=e_{n}^{i}\left(x^{j}-x^{i}\right)-l_{n}^{r e f} \\
& u_{n}=E_{n}^{i}\left(X^{j}-X^{i}\right)(n=1,2,3)
\end{aligned}
$$

$l_{n}^{\text {ref }}$ is reference length. The node rotational equation is as follows.

$$
u_{r 4}=\phi-\phi_{1}^{r e f}
$$

$u_{r 5}=\varphi-\varphi_{2}^{r e f}$

$u_{r 6}=\chi-\chi_{3}^{r e f}$

$u_{r i}(i=4,5,6)$ is three-rotation vector localized under the local coordinate system. $\phi_{1}^{r e f}, \phi_{2}^{r e f}$ and $\phi_{3}^{\text {ref }}$ are reference angles.

The partial beam element model and solid element model need to form a whole by certain assembly methods. In the finite element theory, the node of the beam element has six degrees of freedom, while the node of solid element has only three degrees of freedom. Therefore, the two parts should be connected rigidly. The processing method includes the rigid zone, rigid beam, and constraint equation. Constraint equation can avoid stress concentration which meets plane section assumption at connected section. The node of the beam element is master node, while node of solid element is a slave node. The constraint equation formula is
Const $=\sum_{i=1}^{N}($ Coefficoent $(I) \times U(I))$

Where $U(I)$ is the degree of freedom. Coefficoent $(I)$ is the coefficient of $U(I)$ and $N$ is the equation number.

\section{The fatigue experiment of the integral joint}

\subsection{Project backgrounds}

The Xinghai sea-crossing bridge in Dalian, the first steel truss suspension bridge with double deck seacrossing in China, is taken as an example project. The bridge span is $80 \mathrm{~m}+460 \mathrm{~m}+180 \mathrm{~m}=820 \mathrm{~m}$ and the width is $24 \mathrm{~m}$. The main truss structure is connected by integral joint plate, and all the member sections are a closed box. A main truss is composed of top chord members, lower chord members, vertical web members and inclined web members. Main truss is $10 \mathrm{~m}$ tall, and each standard segment is $10 \mathrm{~m}$ long. The structure of integral joint on top of a chord member is shown in Fig. 2. It is made of Q345steel. The Young's Modulus and shear modulus of Q345 steel are $210 \mathrm{Gpa}$ and 81 Gpa. The Poisson Ratio of Q345 steel is 0.3. The S$\mathrm{N}$ curve of Q345 steel is shown in Fig. 3. As this joint is under multi-direction stress, it has a complicated stress field. It is connected by large numbers of variable welding lines. The traditional single scale structure simulation is unable to analyze the large-scale civil engineering fatigue damage structure.

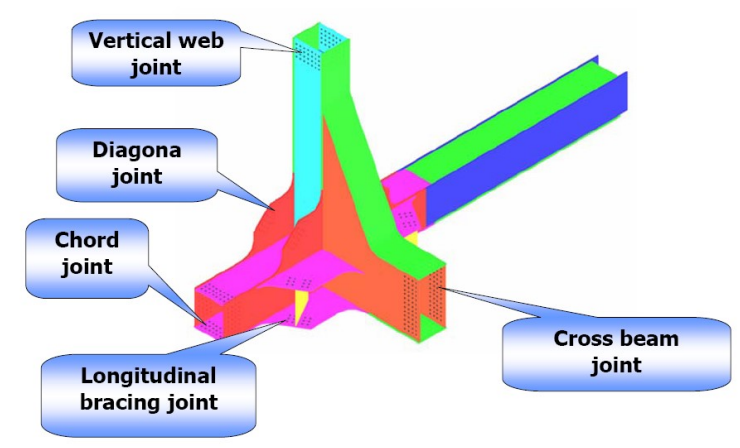

Figure 2. Integral joint of a sea-crossing bridge 


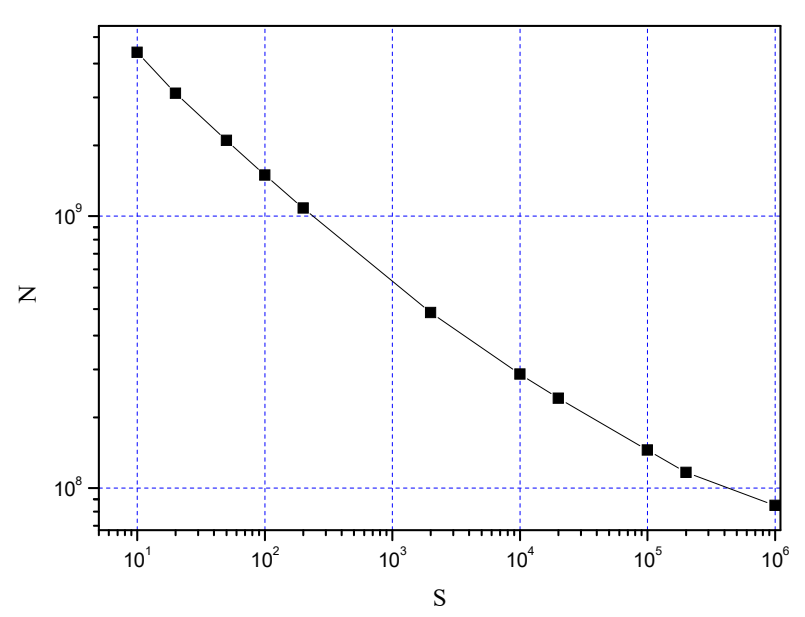

Figure 3. S-N curve of Q345 steel

\subsection{Equivalent fatigue stress amplitude}

Steel bridge fatigue belongs to the variableamplitude low-stress fatigue. According to the current technology conditions, constant amplitude fatigue testing is carried out. Miner's theory is often used to calculate the equivalent stress amplitude $\Delta \sigma_{0}$. The relation between the variable amplitude fatigue strength and the constant amplitude fatigue strength has been established [17].

$\Delta \sigma_{0}=\left[\frac{\sum n_{i}\left(\Delta \sigma_{i}\right)^{m}}{\sum n_{i}}\right]^{1 / m}$

$\Delta \sigma_{i}$ is the $i$-th stress amplitude, $n_{i}$ is the cycle times of $\Delta \sigma_{i}, m$ is material constant. Based on this, British steel bridge design standard BS5400 proposes the following formula.

$\Delta P=\left[K_{F} \frac{\sum n_{i}\left(\Delta P_{i}\right)^{m}}{\sum n_{i}}\right]^{1 / m}$

where $\Delta P$ is the fatigue load amplitude and $K_{F}$ is the multiple traffic lane adjustment coefficient. According to BS5400, we can get $K_{F}=3 . m$ is the slope of S-N curve, generally $m=3 . \Delta P_{i}$ is the internal force amplitude which is caused by one BS5400 standard fatigue vehicle in the $i$-th traffic lane. $n_{i}$ is times that standard fatigue vehicle act on $i$-th traffic lane during 100 years. $n_{0}=2 \times 10^{6}$. After analysis, equivalent fatigue load is $700 \mathrm{KN}$. The arrangement of specimen and strain gauges and strain gauge are shown in Fig. 4 and Fig. 5.

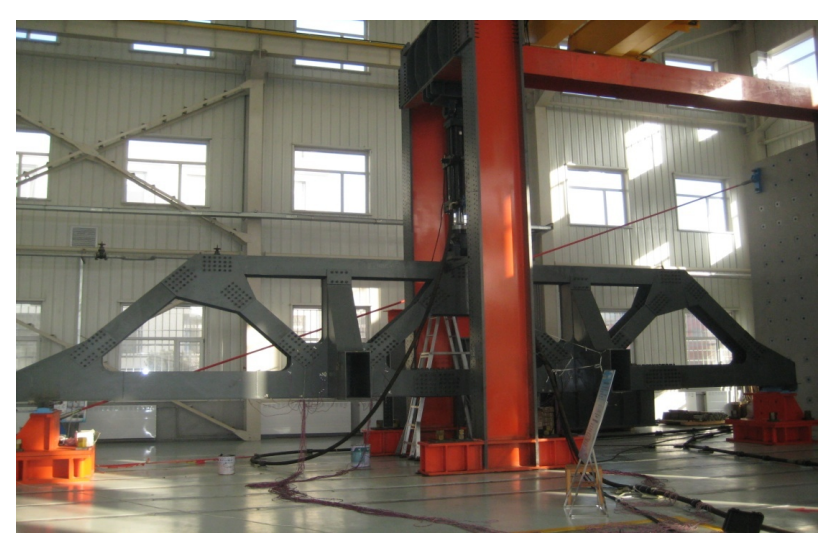

Figure 4. Experimental device of integral joint

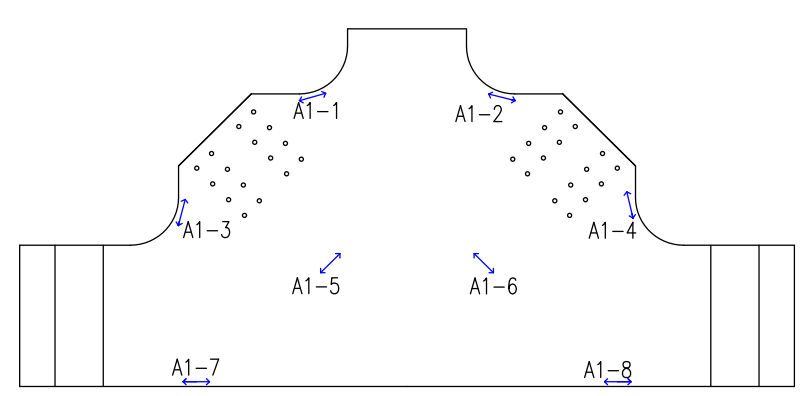

Figure 5. Layout of strain gauges on integral joint

\subsection{Multi-scale FEM numerical simulation}

Structure fatigue failure often occurs with local detail parts so that details of the stress concentration and hot spot stress should be analyzed with solid element in fine size. Then overall structure can accurately calculate the static and dynamic response while small-scale model can simulate fatigue damage. If we adapt solid element to analyze the overall structure, it will be very a complicated analysis. To overcome this problem, we use a multi-scale analysis.

The micro-scales elements are adopted for an integral joint part. The beam elements are adopted for other components. The integral joint part uses solid elements, whereas the connecting component uses ordinary beam elements. The beam elements and micro-scales elements are connected with the connection element. Altogether we establish 4216 solid elements and 16 beam elements. The model 
boundary condition is thus equivalent to fatigue load. $F=450+350 \sin 4 \pi t(\mathrm{KN})$ is applied to node A. Horizontal and vertical restraints are applied to node B and C, as shown in Fig. 6 and Fig. 7. Static loading tests were performed on the fatigue test specimens after every 500,000 cycles of loading. The stress of every point is a linear variation. The result of the point A1-6 is shown in Fig. 8.

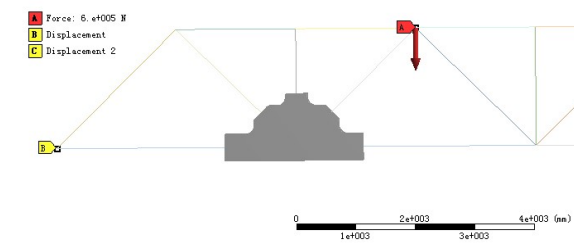

Figure 6. Multi-scale model of overall structure

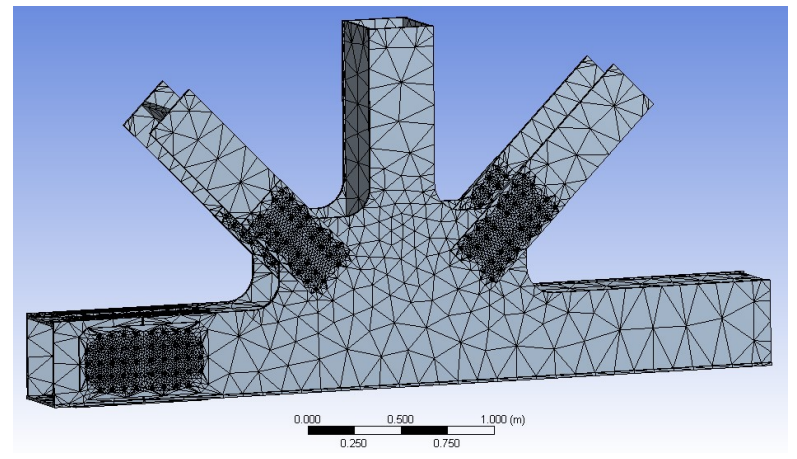

Figure 7. Integral joint model

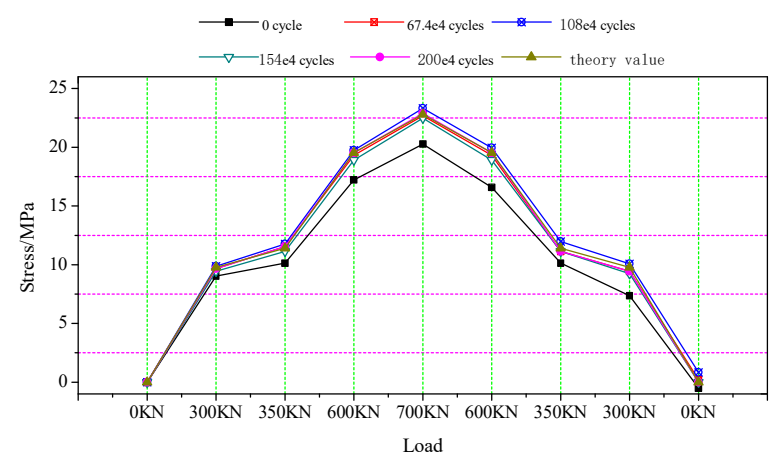

Figure 8. Stepwise loading normal stress change curve of point A1-6

\subsection{Comparing theoretical analysis and experimental results}

The fatigue experiment confirmed the multi-scale FEM. Table 1 shows the stress of all testing joints and the value of the multi-scale FEM analysis after
$2 \mathrm{e} 6$ time loop. The results are close, and the multiscale FEM is therefore reliable.

Based on S-N theory, using Ansys Workbench Fatigue Tools, the fatigue safe factor contour of integral joint is obtained, as shown in Fig. 9.

$$
K=\frac{L_{1}}{L_{2}}
$$

$K$ is a fatigue safe factor, $L_{1}$ is predicted fatigue life, $L_{2}$ is actual fatigue life. Obviously, the integral joint fatigue performance meets fatigue requirements of high quality joints.

Table 1 Theoretical values and the measured stress values after 2e6 time loop of integral joint

\begin{tabular}{cccc}
\hline $\begin{array}{c}\text { Measuring } \\
\text { point }\end{array}$ & $\begin{array}{c}\text { Test } \\
\text { value } \\
\text { /MPa }\end{array}$ & $\begin{array}{c}\text { Analysis } \\
\text { value } \\
\text { /MPa }\end{array}$ & $\begin{array}{c}\text { Test } \\
\text { value/analysis } \\
\text { value }\end{array}$ \\
\hline A1-1 & -15.96 & -14.8 & 1.08 \\
A1-2 & -12.18 & -11.2 & 1.09 \\
A1-3 & 4.2 & 3.5 & 1.20 \\
A1-4 & -24.57 & -5.8 & 4.24 \\
A1-5 & 20.58 & 25.6 & 0.80 \\
A1-6 & 22.89 & 30.8 & 0.74 \\
A1-7 & 22.47 & 35.7 & 0.63 \\
\hline
\end{tabular}

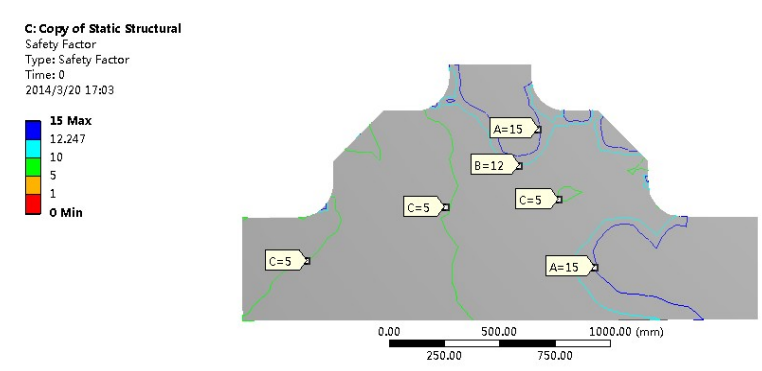

Figure 9. Fatigue safe factor $K$ contour of integral joint

\section{Conclusions}

In multi-scale FEM, a small-scale model with a solid element is adopted to simulate small scale local details and a large-scale model with the beam element is adopted to simulate the overall structure. The boundary conditions of the small- scale model are close to the overall structure. From above it can be concluded that:

(1) The multi-scale FEM can provide an accurate 
simulation of the main research part and ensure the fatigue life forecast so as to simplify calculation. This method is highly efficient and feasible.

(2) The multi-scale FEM and the experimental yields have very close results. The multi-scale FEM provides a new and reliable method for the fatigue performance of structure analysis.

\section{Acknowledgments}

This work is supported by the Fundamental Research Funds for the Central Universities (DUT15QY34); Foundation of State Key Lab of Subtropical Building Science, South China University of Technology (2014KB11); and Foundation of Liaoning Provincial Department of Education Funded Projects (L2014027). The authors would also like to thank the reviewers for their critical comments and valuable feedback which have been very useful in improving the work presented in this paper.

\section{Conflict of Interests}

The authors declare that there is no conflict of interests regarding the publication of this paper.

\section{References}

[1] Babuska, I., Caloz G., Osbom E.: Special finite element methods for a class of second order elliptic problems with rough coefficients. SIAM J Numer Anal., 31 (1994), 4 , 945-981.

[2] Hou Y., Wu, X.-h.: A multiscale finite element method for elliptic problems in composite materials and porous media. J Comp Phy., 134 (1997), 1, 169-189.

[3] Chen Z., Hou, T.: Amixed multiscale finite element method for elliptic problems with oscillating coeffieients, Math Comp, 72 (2002), 541-567.

[4] Allaire, G., Brizzi, R.: Amultiscale finite elementmethod for numerical homogenization. Multiscale Model Simul., 4 (2005), 3, 790-812.

[5] Aarnes, J., Stein, K., Lie, K.: A hierarchical multiscale method for two-phase flow based upon mixed finite elements and nonuniform grids. Multiscale Model Simul., 5 (2006), 2, 337-363.
[6] Zheng, K., Chen, N., Zhang, X.: Research on Technique of Structural Simulation for Entire Bridge, Bridge Construction, 2 (1998), 10-15.

[7] Mi, G., Li, C., Gao, Z., Zhao, D., Niu, J.: Finite element analysis of welding residual stress of aluminum plates under different butt joint parameters, Engineering Review, 34, 2014, 3, 161-166.

[8] Mi, G., Li, C., Chen, L., Xu, L.: Numerical simulation and process optimization on cast steel bearing sleev, Engineering Review, 35 (2015), 1, 19-25.

[9] Sun J.: Multi-scale Analysis of Mechnical Properties on Frecture Process Zone, Xi`an University of Science and Technology, 2009

[10] Qu, W., He, Z., Liu, J.: Analysismethod of fatigue crack propagation and fracture at joints of steel lattice structures under dynamic loads, China civil engineering journal, 43 (2010), 12, 78-86.

[11] Zerbst, U., Beretta, S., Mendez, G. K.: Safe life and damage tolerance aspects of railway axles - A review, Engineering Fracture Mechanics, 98 (2013), 214-271.

[12] Nikishkov, Y., Makeev, A., Seon, G.: Progressive fatigue damage simulation method for composites, International Journal of Fatigue, 48 (2013), 2, 266-279.

[13] Shunpeng, Z., Hongzhong, H., Liyang, X.: Nonlinear Fatigue Damage Cumulative Model and the Analysis of Strength Degradation Based on the Double Parameter Fatigue Criterion, China Mechanical Engineering, 19 (2008), 22, 2753-2758.

[14] Chunsheng, W., Xin, L., Xin, Y., Yue, X.: Fatigue reliability updating evaluation using nondestructive inspections for existing steel bridges, China Civil Engineering Journal, 43 (2010), 8, 81-87.

[15] Ying, W.: Break and fatigue, Wuhan: Chinese Geology University Publishing house, 2008.

[16] Xinmin, W.: ANSYS structure analysis unit with to apply, Beijing, People transportation publishing house, 2011.

[17] Liu, G., Wu, W., Tang, L., Tian-liang, W.: Fatigue Test on Welded Integral Joint of Transverse Truss of Baling River Bridge, Highway, 3 (2010), 3, 96-100. 